\title{
Sector Antenna and DFE Modems for High Speed Indoor Radio Communications
}

\author{
Ganning Yang, Kaveh Pahlavan, and Timothy J. Holt
}

\begin{abstract}
This paper examines the outage probabilities of BPSK and BPSK/DFE radio modems with omnidirectional and six-sector antennas. The effects of room size, data rate, transmitted power and sector antenna patterns on the performance of the systems are analyzed. A 2-D ray tracing algorithm is used to model the radio propagation in a typical indoor environment used for wireless LAN applications. The parameters of the radio propagation model are adjusted to fit the results of simulations to the results of empirical measurements with omnidirectional antenna. It is shown that in a line-of-sight (LOS) environment, a six-sector antenna is more effective than a DFE with an omnidirectional antenna at eliminating the effects of multipath. For an obstructed-line-of-sight (OLOS) environment where at least one wall exists between the transmitter and the receiver, the DFE is more effective than the six-sector antenna. It is shown that a BPSK/DFE modem with a six-sector antenna can support data rates close to $20 \mathrm{Mbps}$.
\end{abstract}

\section{INTRODUCTION}

W IRELESS LAN's demand high data rates in small indoor radio environments which cover several rooms [3], [4], but the maximum data rate in an indoor radio channel is bounded due to the effects of multipath. In order to increase the data rate, performance enhancement techniques such as external diversity, coding, adaptive equalization, and spread spectrum have been studied [1]-[8]. Performance analysis of decision feedback equalizers (DFE) with omnidirectional antennas over the measured and modeled indoor radio channels shows data rates on the order of $10 \mathrm{Mbps}$ for BPSK/DFE modems [1], [5], [6], [8]. Another effective method to increase the data rate is to use a sector antenna [9], however, in the open literature, there are no analytical results for the performance evaluation of indoor radio modems with sector antennas. This paper presents an analytical approach to examine the effectiveness of sector antennas in breaking the data rate limitations of BPSK and BPSK/DFE modems operating in the indoor radio channel.

For a realistic performance evaluation of a modem, measured channel profiles and statistical models based on measured channel profiles are usually used [1], [2] to represent the channel in the calculation of the error rates. However,

Manuscript received February 8, 1993; revised May 12, 1993, November 15, 1993, and March 9, 1994. Part of this paper was presented at the IEEE ICC' 92 in Chicago.

G. Yang is with Rockwell International Corporation, Digital Communications Division, Newport Beach, CA 92658 USA

K. Pahlavan is with the Center for Wireless Information Network Studies, Worcester Polytechnic Institute. Worcester, MA 01609 USA.

T. J. Holt is with Hewlett-Packard, Roseville Networks Division, Roseville, CA 95747 USA.

IEEE Log Number 9403810 all of the available wideband channel measurements and the statistical models have been performed with omnidirectional antennas. These measurements identify all the arriving paths, but do not specify the direction from which the paths arrive. As a result, they can not be used for performance evaluation of systems with sector antennas. A ray tracing algorithm which provides the direction of the paths in an indoor channel must be used instead to model the indoor radio propagation. This paper adjusts parameters of a 2-D ray tracing algorithm for indoor radio propagation to fit the results with those of the empirical measurement, and uses the results of computer simulation for performance prediction of BPSK and BPSK/DFE modems using six-sector antennas.

In dividing an indoor area into microcells, it is desirable to use the existing layout of the building along with a computer program to model the radio propagation. Most existing building layouts are available in 2-D format and, ideally, one would like to scan that layout into the computer and to use a computer program to divide the building into microcells. A ray tracing algorithm is an ideal approach for this application. A 2-D, rather than 3-D, ray tracing algorithm is adopted for four reasons: 1) all available building layouts are 2-D, 2) the simplicity of the 2-D algorithm allows examination of complicated indoor environments, 3) the floor and ceiling features may be highly irregular and filled with furniture and lighting fixtures, thus not appropriately modeled as horizontal planes, and 4) in small indoor areas used in our measurements, the results of 2-D and 3-D simulations showed minor differences, and this conclusion was consistent with the results of other published research [21].

A sector antenna observes the signal arriving from different directions (paths) and selects the one sector with maximum power. Since the signals are arriving from different directions, the sector antenna potentially reduces the effects of multipath, resulting in a higher attainable data rate for the radio modem. A DFE modem uses the signals arriving from different paths to provide implicit or inband diversity [1], [8], which increases the data rate of the system. In the rest of this paper, we will find and compare the effectiveness of the sector antenna, a DFE modem with an omnidirectional antenna, and, finally, a DFE modem with a sector antenna.

Section II provides the details of the channel model and the ray tracing algorithm. It also shows the results of comparisons between simulations using the ray tracing algorithm and actual channel measurements. The method used for performance evaluation is discussed in Section III. Finally, Section IV presents and discusses the results of the performance analysis. 


\section{ChanNel Modeling}

\section{A. Model Description}

The equation used to model the multipath radio channel is a linear filter with an impulse response given by

$$
h(\tau)=\sum_{k=0}^{n} \beta_{k} e^{-j \theta_{k}} \delta\left(\tau-\tau_{k}\right)
$$

where the $\beta_{k}$ 's are real positive gains, the $\theta_{k}$ 's are the phase shifts and the $\tau_{k}$ 's are the propagation delays of all of the channel paths [10]-[12]. The goal of channel modeling is to determine the $\beta_{k}$ 's, $\theta_{k}$ 's and $\tau_{k}$ 's for any transmitter-receiver location in the building.

A physical propagation model of an indoor radio environment consists of several modes: reflection, transmission, diffraction and scattering. In small indoor environments, reflection and transmission are dominant. Diffraction contributes significantly only in cases where rays that are reflected and transmitted through many walls are weaker than diffracted rays, which is the case for small areas used for most WLLAN applications. The irregularity of floor, ceiling, and wall structures causes the phenomena of scattering which causes local variations. It probably does not have significant effects on average, and, in any case, there is no way to precisely determine the effects. The model in this paper considers the effects of specular reflection and transmission, and adjusts the propagation parameters to fit the results with the empirical results.

Determining the specular reflected and transmitted paths is done using geometrical optics theory. Generally, there are two methods that can be employed to calculate these paths. The first uses optical images of the transmitter when the walls are considered as reflectors [15], [16], [18]. The transmitter is reflected and transmitted around the various walls to find all the paths to the receiver. The path distance and direction is then used to determine the magnitude, phase, delay and direction of each arriving path. This method has the advantage that adding the effects of diffraction to the propagation model is relatively easy, and therefore provides better accuracy in large study areas. However, the existence of an image does not imply the existence of a ray path. Therefore, an additional procedure should be adopted to eliminate all the unrealistic paths, and this procedure becomes more difficult in complicated environments.

The second method for determining the paths is through the application of ray tracing techniques [17]-[20], [22]. The ray tracing algorithm is an intuitively simple approach to the problem of multipath propagation. The approach is basically to send a pin cushion of rays from the transmitter and to trace the progress of each ray through the environment until the ray has either intersected the receiver or lost enough power so that its effects are negligible. The time of arrival, magnitude, phase, and direction of arrival is recorded for each ray. This method is accurate for relatively small areas, such as indoor environments. In small indoor areas, the optical imaging and ray tracing techniques provide very close results. The results in this paper are obtained by using the second technique.
When a ray that is being traced hits a wall or some other surface, some of the energy is reflected, some is transmitted through the surface, and some is absorbed. The wall can be modeled as a dielectric slab. Although there are some derivations of reflection and transmission coefficients about a dielectric slab, which are based on the thickness of the slab, the electromagnetic properties of the material, and the frequency of the electromagnetic plane wave, an approximation to these coefficients must be made. This is because the transmitter signal covers a band of frequencies and a wall is a combination of homogeneous, inhomogeneous, and conductive materials; it is not a pure dielectric slab. In this paper, we assume that the reflection coefficient, based on the incident angle and wall material, is determined by the Fresnel formula, and the transmission coefficient is assumed to be determined by the reflection coefficient and the energy loss due to the wall.

If we consider the reflection coefficient as the ratio of electric field strength of the incident ray to the electric field of the reflected ray, and that we have the case that the electric field is parallel to the wall, then the reflection coefficient can be written as

$$
R=\frac{E_{r}}{E_{i}}=\frac{\cos \Omega-\sqrt{n^{2}-\sin ^{2} \Omega}}{\cos \Omega+\sqrt{n^{2}-\sin ^{2} \Omega}}
$$

where $n$ is the complex dielectric constant of reflected material and $\Omega$ is the angle of the incident ray. The transmitted coefficient is assumed as

$$
T=\sqrt{\alpha\left(1-R^{2}\right)}
$$

where $\alpha$ is a power loss due to walls. It is a valid assumption if the frequency range is between $900 \mathrm{MHz}$ and $1.1 \mathrm{GHz}$ and the thickness of wall is about one-third of the wavelength.

Using either one of these deterministic models would allow us to determine the direction of the received ray. This is crucial to the simulation of the sector antenna since we need to know which sector of the antenna receives the signal. In analyzing the performance of sector antennas, we assume that the receiver is equipped with a six-sector directional antenna whose polarizations are vertical. The $i$-th antenna pattern is defined by the function

$$
g_{i}\left(\phi_{k}\right)= \begin{cases}\frac{\sin \left(\frac{2.78\left(\phi_{k}-\frac{\pi i}{3}\right)}{\phi}\right)}{\frac{2.78\left(\phi_{k}-\frac{\pi i}{3}\right)}{\Phi}} & \text { if } \frac{\pi i}{3}-\frac{\Phi}{2} \leq \phi_{k}<\frac{\pi i}{3}+\frac{\Phi}{2} \\ a_{0} & \text { otherwise }\end{cases}
$$

where $g_{i}\left(\phi_{k}\right)$ is the normalized power gain, $\phi_{k}$ is the orientation angle, and $\Phi$ is the $3 \mathrm{~dB}$ beamwidth of the antenna which is around $\frac{\pi}{3}$. A uniform side lobe level $a_{0}$ is assumed to be $10 \mathrm{~dB}$ below the main beam gain if the contributions of the rays received through the sidelobes are considered in the simulation. Thus, the channel impulse response for the $i$-th sector is given by

$$
h_{i}(\tau)=\sum_{k=0}^{n} \beta_{k} e^{-j \theta_{k}} \delta\left(\tau-\tau_{k}\right) g_{i}\left(\phi_{k}\right)
$$

where

$$
\theta_{k}=\frac{2 \pi f_{c} r_{k}}{c}+\sum_{m=0}^{n} \theta_{k m}
$$




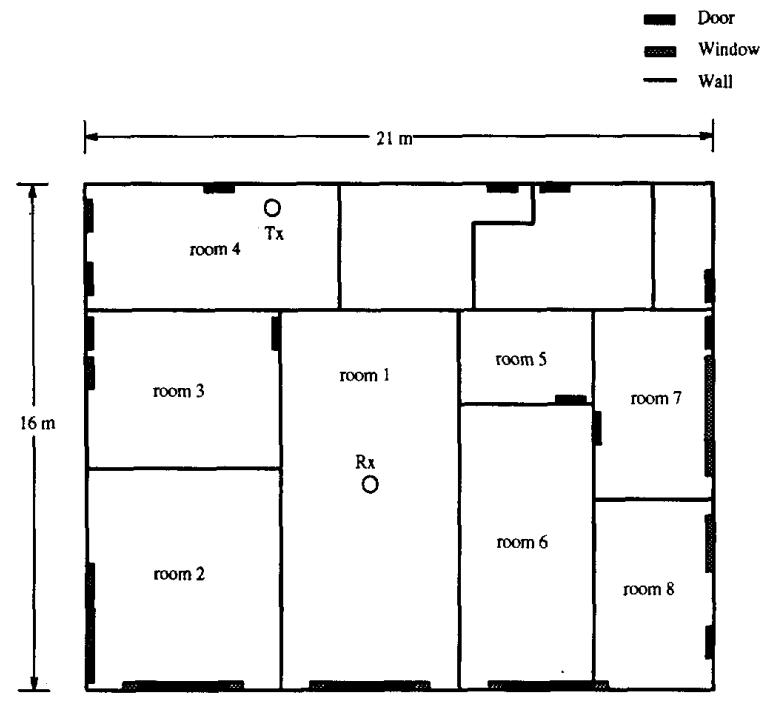

Fig. 1. Floor plan of one section of Atwater Kent 2nd floor.

is the phase of the $k$-th path, $\theta_{k m}$ is $m$-th reflection or transmission phase shift for the $k$-th path and $\phi_{k}$ is the orientation at which the signal is arriving at the receiver. $\beta_{k}$ is the amplitude of the $k$-th path given by

$$
\beta_{k}=\frac{A}{r_{k}} \prod_{m} \alpha_{k m}
$$

where $r_{k}$ is $k$-th path distance, $A$ is the amplitude of received signal at one meter from the transmitter for free space transmission, and $\alpha_{k m}$ is the $m$-th reflectivity or transmission coefficient of the wall for the $k$-th path. This coefficient is determined by the incident angle of the ray and by the material used in the construction of the wall.

\section{B. Comparison with Measurements}

In this paper, the 2-D ray tracing algorithm described above is used to model the indoor radio propagation [20]. The accuracy of the model to fit the statistics of measurements are examined.

Extensive wideband measurements of the second floor of Atwater Kent Laboratory at Worcester Polytechnic Institute are available in the literature [13], [14]. There were approximately 600 wideband measurements in the $0.9 \mathrm{GHz}$ to $1.1 \mathrm{GHz}$ range taken near the center of the building in an area comprised of several laboratories and offices (Fig. 1). The receiver was located at the center of Room 1 and the transmitter was moved to different locations in several of the surrounding rooms. The transmitting and receiving antennas were both $\frac{\lambda}{4}$ monopole antennas with small metal ground planes whose radiation pattern was assumed to be the same as those of a dipole antenna. Both antennas were mounted at $1.6 \mathrm{~m}$ above the floor and the antenna patterns are omnidirectional. The measured received power at $1.0 \mathrm{~m}$ from the transmitter was considered as a reference to calibrate the simulation.

The simplified model of the floor plan that was simulated with the ray tracing program contains all of the internal walls,

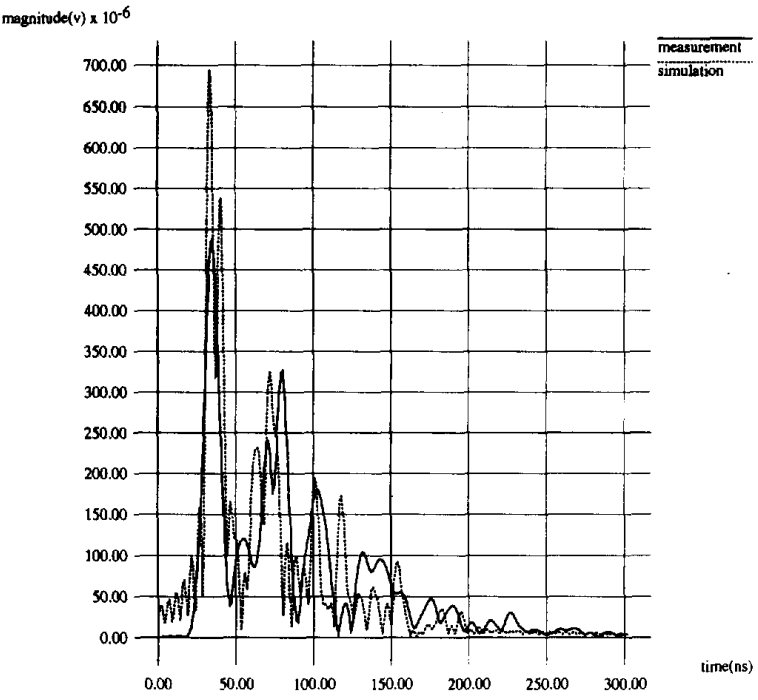

Fig. 2. Channel impulse responses of simulation and measurement in one transmitter-receiver location.

doors and windows in the area. Concrete walls, metal doors, and glass window are treated, respectively, as highly dielectric materials, nearly perfect conductors, and low dielectric materials and are all treated separately in the calculation of the reflection and transmission coefficients. The permittivity, conductivity and power loss due to walls are selected to fit the received power and delay spread with the empirical results. The permittivity and conductivity of the walls are assumed to be 15.0 and 0.001 , respectively, whereas the permittivity and conductivity of the glass windows are assumed to be 3.0 and 0.001 , respectively. When a ray penetrates a wall or window, the energy loss due to irregularity is assumed to be 0.2 . The reflection and transmission coefficients of the metal doors are assumed to be 0.95 and 0.0 , irrespective of the angle of incidence.

The channel impulse responses for the transmitter-receiver pair marked in Fig. 1 for both the simulation and the measurements are shown in Fig. 2. The received powers of simulated and measured are $-49.9 \mathrm{~dB}$ and $-54.4 \mathrm{~dB}$, respectively, while the RMS delay spreads are $20.7 \mathrm{~ns}$ and $23.6 \mathrm{~ns}$. From this figure, it is shown that ray tracing predicts the major paths fairly well. However, it should be noted that site to site comparison may be sensitive due to complicated indoor environments. Thus, statistical characteristics of channels over a particular area are important for estimating the coverage of radio systems, especially when the results are used for performance evaluation of the systems. In the following simulations, the statistical characteristics of the radio channels will be compared with the measurements. For each simulation, the RMS delay spread of the channel and the received power was calculated. All of the values for power measurements have been normalized to the mean value of received $\mathrm{dB}$ power for the entire floor plan.

In the first simulation, the receiver was placed at over 1300 evenly spaced locations in the floor plan (UNIFORM). The 


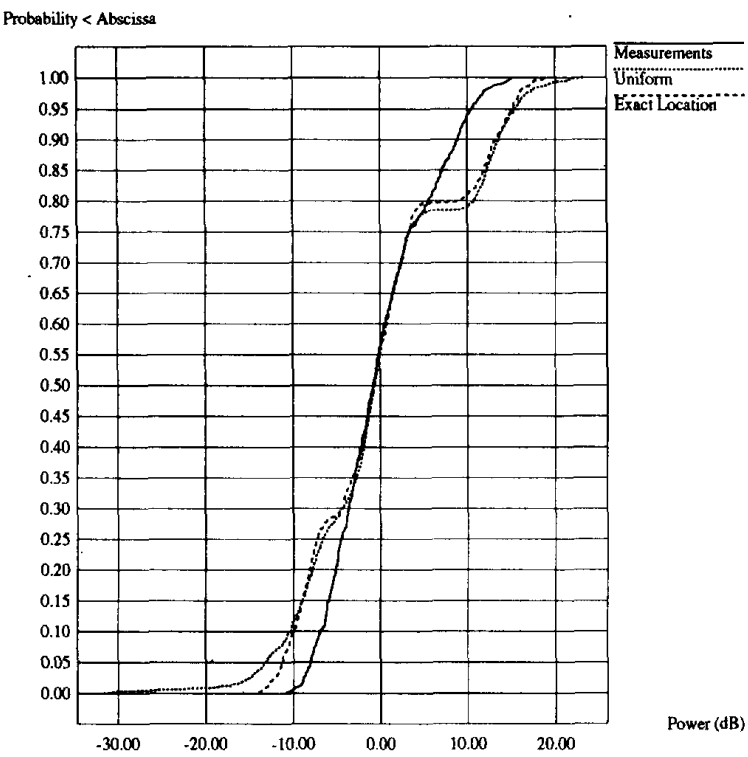

Fig. 3. CDF of the relative received powers in AK 2 nd floor.

CDF's of the normalized received power and RMS delay spread are shown in Figs. 3 and 4, respectively. These two figures also show the measured received power and RMS delay spread. The second simulation involved placing the receiver at the same locations that were used in the measurements (EXACT). The received power and RMS delay spread from this simulation are also shown in Figs. 3 and 4. To check the accuracy of the 2-D ray tracing versus 3-D model, the RMS delay spread and the power of selected points in the area obtained from two models were compared. The mean and standard deviation of the difference between the powers determined from the two models averaged over the sample points were $0.7 \mathrm{~dB}$ and $0.9 \mathrm{~dB}$. The cumulative distribution functions of the RMS delay spreads obtained from the two models were almost the same. Since the statistics of the power and RMS multipath spread of the 2-D and 3-D models are very close, the conclusions obtained from performance evaluation of the modems using either of the models remains the same.

Using the results of UNIFORM and EXACT 2-D simulations, the data was broken up into individual rooms. The normalized received power and RMS delay spread in Room 2, for both the measurements and the two simulations, are shown in Figs. 5 and 6. The results are fairly accurate for Room 1 and Room 2, but for the rooms farther away (Room 7 and Room 8), the results are less accurate but still within reasonable bounds (see Figs. 7 and 8). Table I shows the average and standard deviation of the normalized received powers and RMS delay spreads for each room in the floor plan. This table shows both the measured values as well as the results from the second simulation where the receiver was placed at the same locations that were measured. The reason for a discrepancy between measurement and simulation in Room 7 and Room 8 is that some paths with significant energy but fairly long delay travel along the hallway, which is not included in the simulated floor plan. Thus, a more complete floor plan may be required to

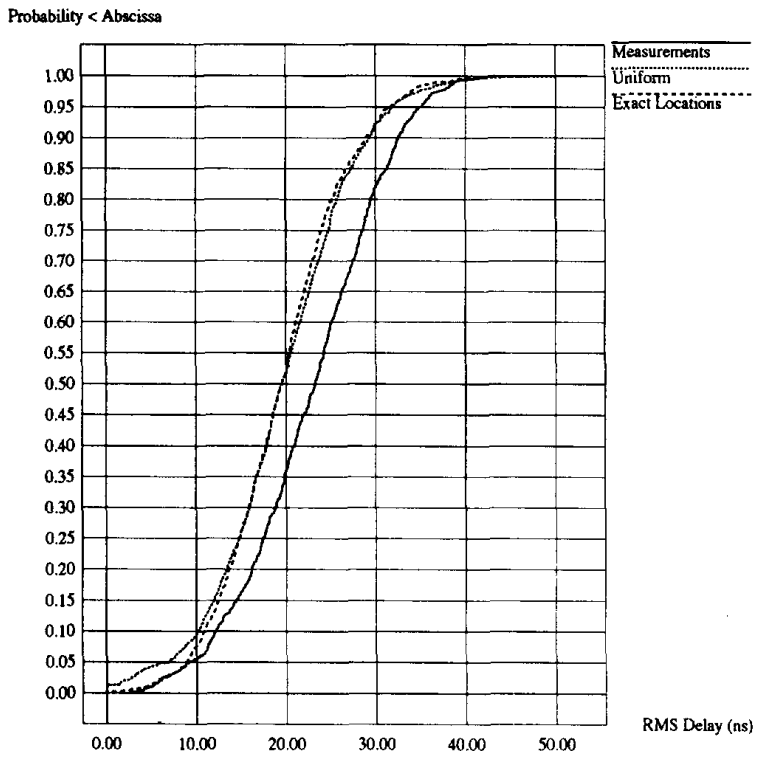

Fig. 4. CDF of the RMS delay spreads in AK 2nd floor.

TABLE I

The Mean and Standard Deviation of the Normalized Received POWER AND RMS DElay SPREAD IN EACH ROOM OF AK 2ND Floor for BOTh the Simllation and tHE MEASlirements

\begin{tabular}{|c||c|c|c|c|c|c|c|c|}
\hline \multicolumn{2}{|c|}{ Mean and Standard Deviation of Power and RMS Delay Spread } \\
\hline \hline \multicolumn{1}{||||}{} & \multicolumn{3}{c|}{ Relative Received Power (dB) } & \multicolumn{3}{c|}{ RMS Delay Spread (ns) } \\
\cline { 2 - 10 } & Measurement & \multicolumn{2}{c|}{ Simulation } & \multicolumn{3}{|c|}{ Measurement } & \multicolumn{2}{|c|}{ Simulation } \\
\hline \hline Room & Mean & S.D. & Mean & S.D. & Mean & S.D. & Mean & S.D. \\
\hline 1 & 8.5 & 2.8 & 13.4 & 2.2 & 15.7 & 7.2 & 14.0 & 6.5 \\
\hline 2 & 1.0 & 2.9 & 0.4 & 1.9 & 22.4 & 6.9 & 20.2 & 6.5 \\
\hline 3 & 1.2 & 3.1 & -1.5 & 2.1 & 21.5 & 8.0 & 22.6 & 5.3 \\
\hline 4 & -4.5 & 1.7 & -6.5 & 4.0 & 26.0 & 7.0 & 27.2 & 7.5 \\
\hline 5 & -2.9 & 1.7 & -2.6 & 1.5 & 24.8 & 5.9 & 20.8 & 2.9 \\
\hline 6 & 0.6 & 2.3 & 2.0 & 1.5 & 21.3 & 7.4 & 19.9 & 4.2 \\
\hline 7 & -7.2 & 1.8 & -10.3 & 1.8 & 29.2 & 6.3 & 15.2 & 2.3 \\
\hline 8 & -5.3 & 1.7 & -8.1 & 0.7 & 24.6 & 6.9 & 19.4 & 3.4 \\
\hline
\end{tabular}

improve the accuracy of predictions in Room 7 and Room 8. Looking at the results of the average received power in each room, we can see that in the simulation the mean power in Room 1 is almost $5 \mathrm{~dB}$ higher than the measured mean power. This is because the received power of each location in the floor plan has been normalized to the mean of all the data points in $\mathrm{dB}$. In this simulation, the outer rooms probably received too little power due to the selection of a large permittivity to provide a better fit for the RMS delay spread.

\section{Performance Analysis}

For a given overall channel impulse response, a unique derivation provides the probability of error calculations for a BPSK modem [23]. With simple modifications, these equations can be used for sector antennas, as well as for BPSK/DFE modems. We will first explain the method used for the performance prediction of a BPSK modem with omnidirectional 


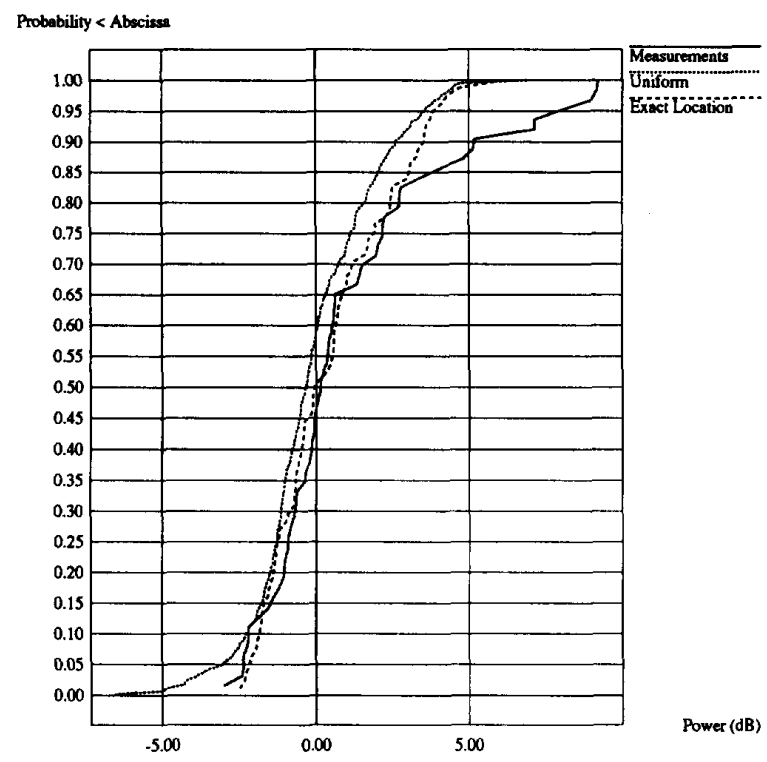

Fig. 5. CDF of the relative received powers in Room 2 of AK 2nd floor.

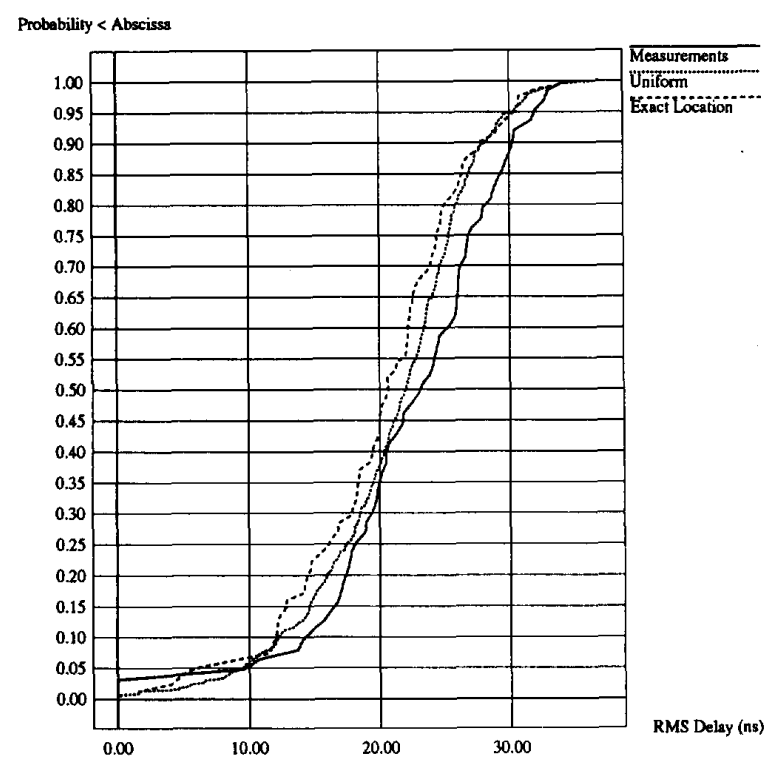

Fig. 6. CDF of the RMS delay spreads in Room 2 of AK 2nd floor.

antennas in which the overall impulse response is represented by (1). We will then extend the results for the case of sector antennas and BPSK/DFE modems.

To make the analysis and simulations tractable, several assumptions are made. Error propagation in the DFE analysis is not treated, perfect clock and carrier recovery is assumed, the sector selection algorithm is assumed to work perfectly, and the phase jumps associated with sector selection steps are assumed to have no impact on carrier recovery. Calculation of the probability of error for a BPSK modem operating over a channel with multipath distortion requires specification of the timing and phase recovery circuits. The timing instant which

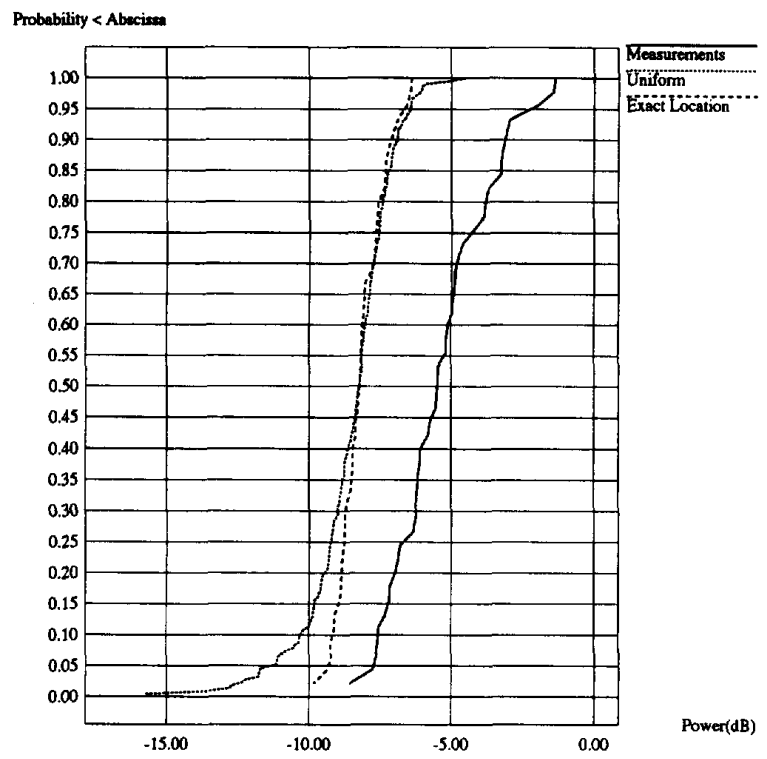

Fig. 7. CDF of the relative received powers in Room 8 of AK 2nd floor.

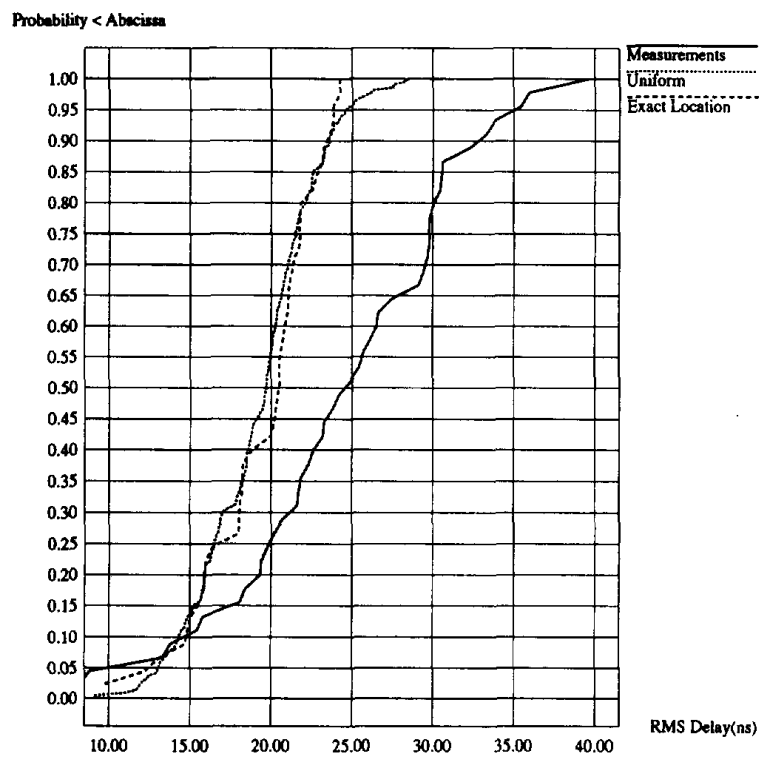

Fig. 8. CDF of the RMS delay spreads in Room 8 of AK 2nd floor.

depends on the time-variation of the multipath channel is a crucial parameter for the system performance [24], [25]. The derivation of this parameter in indoor radio channels follows the analysis in [26]. In this analysis, the timing instant is given by

$$
t_{d}=-\frac{T}{2 \pi} \tan ^{-1} \xi
$$

where $T$ is the symbol interval and $\xi$ is the phase of $D_{1}=$ $\left|D_{1}\right| e^{-j \xi}$, the power in the first harmonic of the received signal 
[25]. The parameter $D_{1}$ is given by

$$
D_{1}=C \sum_{k=0}^{n} \sum_{l=0}^{n} \tilde{\beta}_{k} \tilde{\beta}_{l}{ }_{l}^{*} \frac{\sin \frac{\pi \alpha\left(\tau_{k}-\tau_{l}\right)}{T}}{\frac{\pi \alpha\left(\tau_{k}-\tau_{l}\right)}{T}} \frac{e^{-j \frac{\pi\left(\tau_{k}+\tau_{l}\right)}{T}}}{1-\frac{\alpha^{2}\left(\tau_{k}-\tau_{l}\right)^{2}}{T^{2}}}
$$

where $C$ is a constant which has no effect on the $t_{d}$ and $\alpha$ is the roll-off factor of the raised cosine filter used for pulse shaping. The parameter $\tilde{\beta}_{k}=\beta_{k} e^{-j \theta k}$, in which $\beta_{k}$ 's, $\theta_{k}$ 's, $\tau_{k}$ 's are given by (1).

For the calculation of the carrier recovery, the near optimum remodulation scheme suggested in [27] is used. In this method, the phase of the overall complex baseband channel impulse response is used as the phase reference. The overall complex baseband impulse response of the channel is the convolution of the channel impulse response $h_{i}(t)$ given by (1) and the raised cosine pulse shape $P(t)$. If it is written as

$$
P(t)=P_{R}(t)+j P_{I}(t)
$$

the carrier phase is given by

$$
\gamma=\tan ^{-1}\left[\frac{P_{I}(t)}{P_{R}(t)}\right]
$$
is

Given the carrier and timing recovery, the decision variable

$$
S\left(t_{d} \mid\left\{a_{n}\right\}\right)=\sum_{n} a_{n} \operatorname{Re}\left[P\left(t_{d}-n T\right) e^{-j \gamma}\right]
$$

where $\left\{a_{n}\right\}$ is the transmitted information sequence. The probability of error for a BPSK system is given by [23]

$$
P(e)=\frac{1}{2} \sum P\left(\left\{a_{n}\right\}\right) \operatorname{erfc}\left(\frac{S\left(t_{d} \mid\left\{a_{n}\right\}\right)}{\sigma_{N}}\right)
$$

where $\sigma_{N}$ is the variance of the additive noise and

$$
\operatorname{erfc}(x)=\frac{2}{\sqrt{\pi}} \int_{x}^{\infty} e^{-t^{2}} d t .
$$

To extend these calculations to the sector antenna system, the same equations are used, (5) rather than (1) representing the channel impulse response. For the BPSK/DFE modems [1], the overall sampled impulse response after equalization is used instead of $P\left(t_{d}-n T\right)$ in (11).

\section{RESULTS AND DISCUSSIONS}

In the indoor environment, the radio channels are timeinvariant if no objects are moving, or if we ignore the effects of moving objects. Statistics of spatial variations of the BER over a specific area are very important for the installation of wireless products and estimation of the coverage. The outage probability is estimated as the fraction of locations in the area with unacceptable error rates.

In the following analysis, BPSK and BPSK/DFE radio modems with omnidirectional and six-sector antennas are considered. For the DFE, the minimum mean square error criterion is used to determine the coefficients of the forward and feedback taps of the equalizer. The DFE uses a fractionally spaced (T/2) forward equalizer to eliminate the sensitivity to the sampling time and the need for an accurate front end filter matched to the transmitted pulses. For the sector antenna, six

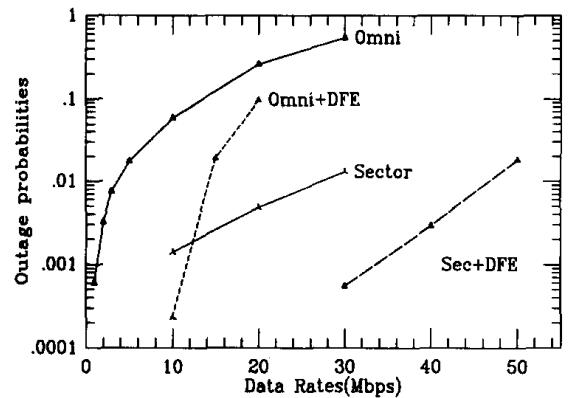

Fig. 9. Outage probabilities versus data rates for four kinds of modems in $30 m \times 30 m$ room.

sectors are used and the sector selection criterion is the sector with maximum power. It is true that other selection criteria may result in better performance, but they are more difficult to implement.

To compare the performance of omnidirectional and sector antennas, the received unfaded carrier-to-noise ratio (CNR) at one meter distance from the transmitter is assumed to be the same for both antennas. As an example for calculation of the unfaded CNR, assuming that the received bandwidth $B$ is $10 \mathrm{MHz}$, the temperature $\mathrm{T}$ is $290 \mathrm{~K}\left(17^{\circ} \mathrm{C}\right)$, transmitter power is $100 \mathrm{mw}$, and the received front end noise is $9 \mathrm{~dB}$. Then, the thermal noise, $\mathrm{kTB}$, is $-104 \mathrm{dBm}[28]$ at the received antenna, and then the noise level is $-95 \mathrm{dBm}$. If attenuation at one meter from the transmitter is assumed to be $35 \mathrm{~dB}$, then the unfaded CNR will be $80 \mathrm{~dB}$ for both omnidirectional and sector antennas.

\section{A. Performance Prediction in an LOS Environment}

In office environments, there are often large, open, inner areas. A simple square room LOS environment is used to compare the performance of the four types of modems being examined. The receiver is assumed to be located at the center of the room and the transmitter is located at many different locations throughout the room.

Fig. 9 illustrates outage probabilities for systems with different modems versus the data rates in a $30 \mathrm{~m} \times 30 \mathrm{~m}$ room. The transmitter power is assumed to be $100 \mathrm{~mW}$. This figure illustrates that if a system with an omnidirectional antenna is used in a $30 \mathrm{~m} \times 30 \mathrm{~m}$ room, the data rate can only reach 3 Mbps with an outage rate of 0.01 for an acceptable error rate of $10^{-5}$. If a six-sector antenna system is used, and the selection criterion is the sector with the highest power, a data rate of $20 \mathrm{Mbps}$ can be achieved, whereas a system with a DFE can only reach a data rate of less than $15 \mathrm{Mbps}$. A DFE with three forward taps and three feedback taps is successful at a data rate of around $10 \mathrm{Mbps}$, but seems to be unsuccessful for a data rate of above $15 \mathrm{Mbps}$. Systems with sector antennas and a DFE can work at a data rate of up to $40 \mathrm{Mbps}$.

Fig. 10 represents outage probabilities versus unfaded CNR at one meter from the transmitter given different data rates for a BPSK modem with sector antennas in a $30 \mathrm{~m} \times 30 \mathrm{~m}$ room. It is found that the probabilities of outage show little sensitivity 


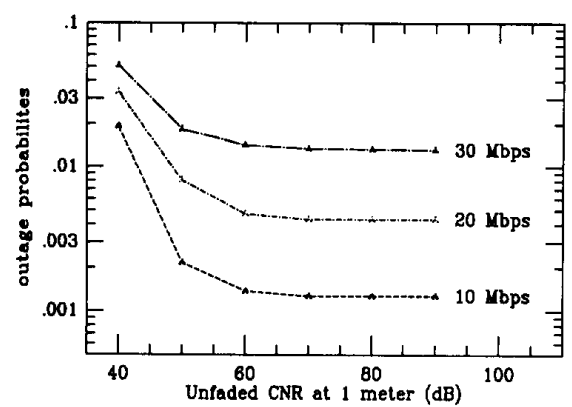

Fig. 10. Outage probabilities versus unfaded CNR at one meter from the transmitter for a BPSK modem with sector antennas.

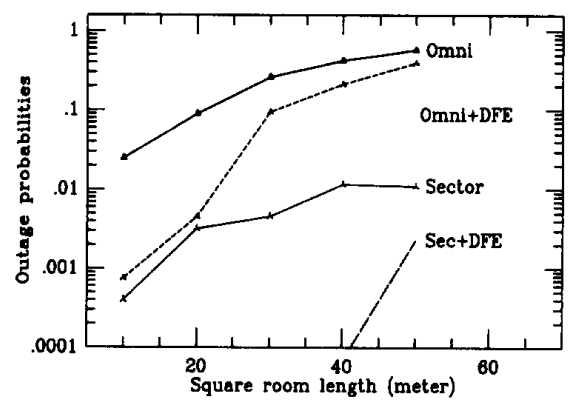

Fig. 11. Outage probabilities versus room size for different modems.

with respect to unfaded $\mathrm{CNR}$ when the transmitter power level is in the range of high unfaded CNR.

Fig. 11 represents the probabilities of outage for different modems with a transmitter power of $100 \mathrm{~mW}$ at a data rate of $20 \mathrm{Mbps}$ versus the room size. It is hard to conclude whether the sector antenna or the DFE has a better performance in a square room smaller than $20 \mathrm{~m} \times 20 \mathrm{~m}$. Better performance will be achieved, however, if the sector antenna is used in a room larger than $30 \mathrm{~m} \times 30 \mathrm{~m}$.

Fig. 12 shows four different antenna patterns and Fig. 13 gives outage probabilities versus data rate limitations for a BPSK modem with different sector antennas based on the nonoverlapped antenna span pattern (I), the optimum antenna span pattern (II) without sidelobes, and the ideal pattern (III) in a $30 m \times 30 m$ room. This figure shows that the sector antenna patterns have significant effects on the performance of the modem and that a $10 \mathrm{~dB}$ difference in the transmit power causes little effect on the outage probability. It also provides limitation of performance gain from antenna patterns.

Fig. 14 gives outage probabilities versus data rate limitations for a BPSK modem with different sidelobes and the level of sector antennas based on optimum antenna span patterns (IV). It is shown that effects of sidelobes of sector antennas on the outage probability is negligible if the sidelobe level is more than $10 \mathrm{~dB}$ below the main beam gains.

\section{B. Performance Prediction in an OLOS Environment}

The above simulations show that using a sector antenna is an effective technique to counteract the harmful effects of

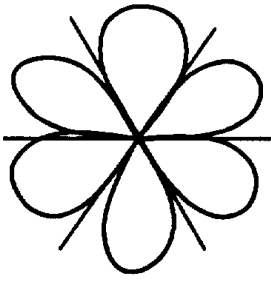

(a)

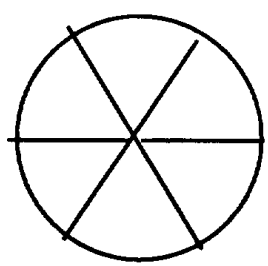

(c)

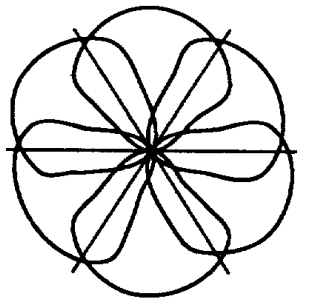

(b)

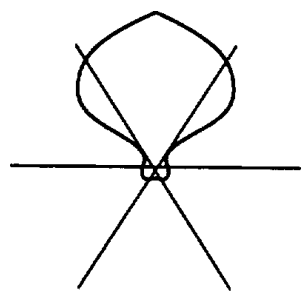

(d)
Fig. 12. Four different antenna patterns. (a) Nonoverlapped pattern. (b) Optimum pattern without sidelobes. (c) Ideal pattern. (d) Optimum pattern with sidelobes (one sector).

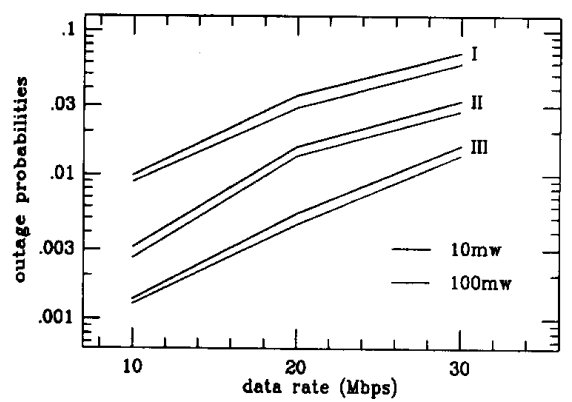

Fig. 13. Effects of antenna patterns for a BPSK modem with sector antennas. (I) Nonoverlapped span pattern. (II) Optimum span pattern without sidelobes. (III) Ideal pattern.

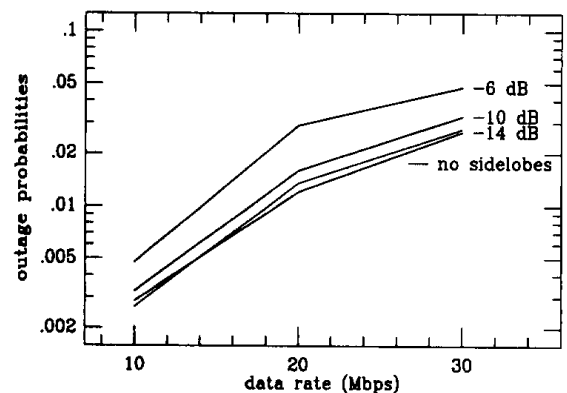

Fig. 14. Effects of sidelobe levels for a BPSK modem with sector antennas based on optimum span patterns. The transmitted power is $100 \mathrm{MW}$ and the room size is $30 \mathrm{~m} \times 30 \mathrm{~m}$.

multipath. In this section, the sector antenna and the DFE are analyzed in an OLOS environment. The floor plan that was analyzed in Section II-B is used here (see Fig. 1). The receiver is located at the center of Room 1 and the transmitter is moved to different locations in the floor plan. In order to 
TABLE II

The Maximum Data Rates for Different Modems in Different Rooms. The Criterion is that if the Error Rate Threshold is $10^{-5}$

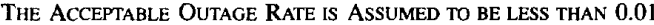

\begin{tabular}{|c|c|c|c|c|}
\hline \multicolumn{5}{|c|}{ Maximum Data Rate (Mbps) } \\
\hline \hline Room & Omni & Omni+DFE & Sector & Sector+DFE \\
\hline 1 & 8 & 40 & 50 & 50 \\
\hline 2 & 5 & 20 & 12 & 25 \\
\hline 3 & 4 & 15 & 13 & 20 \\
\hline 4 & 2 & 7 & 9 & 13 \\
\hline 5 & 4 & 20 & 20 & 40 \\
\hline 6 & 7 & 25 & 30 & 50 \\
\hline 7 & 2 & 6 & 8 & 12 \\
\hline 8 & 2 & 7 & 8 & 15 \\
\hline \hline overall & 3 & 12 & 10 & 18 \\
\hline
\end{tabular}

obtain the outage probabilities, the number of simulations in each room is around 5000. The maximum data rates for four different kinds of modems in each room and for the overall floor plan are obtained.

From Table II, it can be seen that the average maximum data rate over the entire floor plan is $12 \mathrm{Mbps}$ for a BPSK/DFE modem with an omnidirectional antenna and $10 \mathrm{Mbps}$ for a BPSK modem with a sector antenna. The DFE's with three forward taps and three feedback taps are slightly better than the six-sector antennas. In a small LOS environment such as Room 1 , high data rates can be achieved using each technique. If the receiver moves to one of the adjoining rooms, such as Rooms $2,3,5$ or 6 , the BPSK/DFE modem with omnidirectional antennas can still attain a data rate above $15 \mathrm{Mbps}$. However, the maximum data rate for the BPSK modem with the sector antennas in Room 3 drops to 12 Mbps. In Rooms 4, 7 and 8, only the BPSK/DFE with sector antennas can achieve a data rate of above $10 \mathrm{Mbps}$, and sector antennas are slightly better than DFE's.

The results of Table II seem to be overly optimistic but are, in fact, quite reasonable. There are several possible reasons for this. The entire floor plan is confined to a relatively small area in which the maximum radius is about $10 \mathrm{~m}$, the RMS delay spread is less than $30 \mathrm{~ns}$, and the received power is not exceptionally low in any location. The results from Room 1 show that any modem can achieve high data rates. In reality, this is not true since the effects of phase jitter and timing shift will be dominant.

The worst performance is achieved in Room 7. Fig. 15 shows that if the data rate is below $12 \mathrm{Mbps}$, the performance of the BPSK modem with sector antennas is better than a BPSK/DFE modem with an omnidirectional antenna. However, the sector antenna seems to be less effective for a data rate higher than $15 \mathrm{Mbps}$, in this worst case. The BPSK/DFE modem with sector antennas obtains relatively little gain over the BPSK/DFE modem with omnidirectional antennas at very high data rates.

\section{CONCLUSIONS}

A 2-D ray tracing algorithm was used for analyzing the performance of sector antenna systems in the small indoor radio areas typically used for WLLAN applications. The

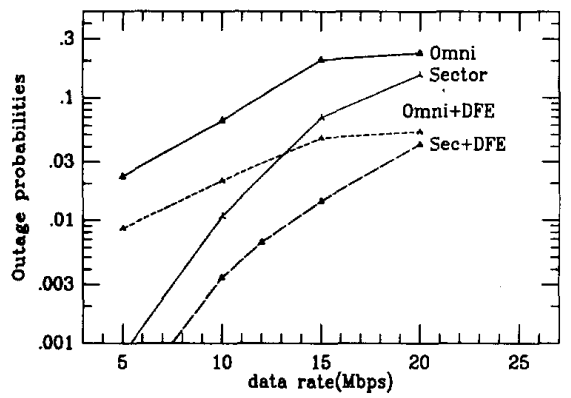

Fig. 15. Outage probabilities versus data rates for four kinds of modems in Room 7.

parameters of the channel model were adjusted by comparing the results of computer simulations to the results of actual wideband measurements. Based on this channel model, a comparative performance evaluation of BPSK and BPSK/DFE radio modems with omnidirectional and six-sector antennas was given in both a simple LOS environment and a more complex OLOS environment, upon the same assumptions of the analysis and simulations stated in Section III. In a large simple room (LOS), extensive computer simulation showed that using a six-sector antenna is a very effective technique to counteract the harmful effects of multipath, and that it is a better technique than using a DFE. This can be explained by the fact that there exists an LOS path, and it may be easy for sector antenna to clarify the delay profile. In a more practical (OLOS) environment, the layout of the rooms in the floor plan has significant effects on the performance of the different modems. Therefore, the conclusions made here are only valid for the similar areas. It was shown that, over the entire floor plan, the DFE achieved slightly better performance than the sector antenna but that the sector antenna seems to be more resistant to the worse case environment than the DFE. Although a combination of a DFE and a sector antenna seems to achieve higher data rates than other modems, the tradeoff between the efficiency and the complexity needs to be considered.

\section{ACKNOWLEDGMENTS}

The authors would like to thank Dr. S. J. Howard for his help with the channel measurements in the Atwater Kent building, and Professor Jin-Fa Lee for his help with the ray tracing algorithm.

\section{REFERENCES}

[1] K. Pahlavan, S. Howard and T. Sexton, "Decision feedback equalization of the indoor radio channel," IEEE Trans. Commun., vol. 41, no. 1, pp. 164-170, Jan. 1993.

[2] M. Chase and K. Pahlavan, "Performance of spread spectrum multiple access over measured indoor radio channels," Proc. IEEE ICC, June 1991.

[3] K. Pahlavan, "Wireless communication for office information net works," IEEE Commun. Mag., vol. COM-23, pp. 19-27. June 1985.

[4] "Wireless infraoffice networks," ACM Trans. Office Inform Syst., vol. 6, pp. 277-303, July 1988.

[5] C. L. Despins, D. D. Falconer and S. A. Mahmoud, "Compound strategies of coding, equalization and space diversity for wideband 
TDMA indoor wireless channels," IEEE Trans. Veh. Technol,, vol. 41, pp. 369-379, Oct. 1992.

[6] C. L. Despins, "Coding and optimum baseband combining for wideband TDMA indoor wireless channels," Ph.D. dissertation, Carleton Univ., Ottawa, Canada, Aug. 1991.

[7] K. Pahlavan and M. Chase, "Spread spectrum multiple access performance of orthogonal codes for indoor radio communications," IEEE Trans. Commun., vol. 38, pp. 574-577, May 1990.

[8] T. A. Sexton and K. Pahlavan, "Channel modeling and adaptive equalization of indoor radio channels," IEEE J. Select. Areas Commun., vol. 7, pp. 114-121, Jan. 1989.

[9] T. A. Freeburg, "Enabling technologies for wireless in-building network communications-Four technical challenges, four solutions," IEEE Commun. Mag., vol. 29, pp. 58-64, Apr. 1991.

[10] A. A. M. Saleh and R. A. Valenzuela, "A statistical model for indoor multipath propagation," IEEE J. Select. Areas Commun., vol. SAC-5, no. 2, pp. 128-137, Feb. 1987.

[11] R. Ganesh and K. Pahlavan, "Modeling of the indoor radio channel," IEE Proc. I, June 1991

[12] S. J. Howard and K. Pahlavan, "Autoregressive modeling of wideband indoor radio propagation," IEEE Trans. Commun., vol. 40, no. 9, pp. $1540-1552$, Sept. 1992

[13] K. Pahlavan and S. J. Howard, "Frequency domain measurements of the indoor radio channel," Electron. Lett., vol. 25, pp. 1645-1647, Nov. 1989.

[14] S. J. Howard and K. Pahlavan, "Measurement and analysis of the indoor radio channel in the frequency domain," IEEE Trans. Instrum. Meas., vol. 39 , no. 5, pp. 751-755, Oct. 1990.

[15] A. J. Rustako, Jr., N. Amitay, G. J. Owens, and R. S. Roman, "Radio propagation at microwave frequencies for line-of-sight microcellular mobile and personal communications," IEEE Trans. Veh. Technol., vol. 40, no. 1, pp. 203-210, Feb. 1991.

[16] J. W. McKown and R. L. Hamilton, Jr., "Ray tracing as a design tool for radio networks," IEEE Network Mag. vol. 5, pp. 27-30, Nov. 1991.

[17] G. A. Deschamps, "Ray techniques in electromagnetics," Proc. IEEE, vol. 60 , no. 9, pp. 1022-1035, Sept. 1972.

[18] M. C. Lawton, R. L. Davies and J. P. McGeehan, "A ray launching method for the prediction of indoor radio channel characteristics," in Proc. IEEE Int. Symp. Personal, Indoor and Mobile Radio Communications, King's College London (U.K.), pp. 104-108, Sept. 1991.

[19] F. Ikegami, T. Takeuchi and S. Yoshida, "Theoretical prediction of mean field strength for urban mobile radio," IEEE Trans. Antenn. Propagat., vol. 39, no. 3, pp. 299-302, Mar. 1991.

[20] T. Holt, K. Pahlavan, and J. F. Lee, "A graphical indoor radio channel simulator using 2-D ray tracing," presented at the IEEE Int. Symp. Personal, Indoor and Mobile Radio Communications, Boston, MA, Oct. 1992.

[21] W. Honcharenko, H. L. Bertoni, J. L. Dailing, J. Qian, and H. D. Yee, "Mechanisms governing UHF propagation on single floors in modern office buildings," IEEE Trans. Veh. Technol., vol. 41, no. 4, pp. 496-504, Nov. 1992.

[22] T. S. Rappaport and D. A. Hawbaker, "A ray tracing technique to predict path loss and delay spread inside buildings," in IEEE GLOBECOM" 92 , Orlando, FL, pp. 649-653, Dec. 1992.

[23] K. Pahlayan "Comparison between the performance of QPSK, SQPSK, QPR, and SQPR systems over microwave LOS channels," IEEE Trans. Commun., vol. COM-33, pp. 291-296, Mar. 1985.

[24] J. C. Chuang, "The effects of multipath delay spread on timing recovery," IEEE Trans. Veh. Technol. vol. VT-35, no. 3, pp. 135-140. Aug. 1987

[25] N. Amitay and L. J. Greenstein, "Multipath outage performance of digital radio receivers using finite-tap adaptive equalizers," IEEE Trans. Commun., vol. COM-32, pp. 597-608, May 1984

[26] R. A Valenzuela "Performance of quadrature amplitude modulation for indoor radio communications," IEEE Trans. Commun., vol. COM-35. no. 11 , pp. 1236-1238, Nov. 1987.

[27] L. J. Greenstein and B. A. Czekaj-Augun, "Performance comparisons among digital radio techniques subjected to multipath fading," IEEE Trans Commun, vol. COM-30, pp. 1184-1197, May 1982.

[28] W. C. Y. Lee, Mobile Cellular Telecommunications Systems. New York: McGraw-Hill, 1989.

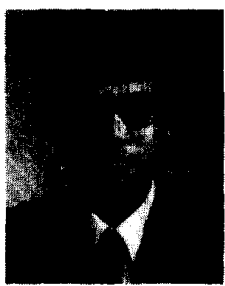

Ganning Yang was born in Nanjing, China on April 6, 1963. He received the B.S. and M.S. degrees from the University of Science and Technology of China, Hufei, China, in 1986 and 1989, and the Ph.D. degree from Worcester Polytechnic Institute MA in 1994, respectively, all in electrical engineering.

Following graduation, he joined Rockwell In ternational Corporation to begin work on wireless communication systems development. His research interests are in performance analysis of wireles communication systems, radio propagation, and channel modeling in indoor or mobile radio channels.

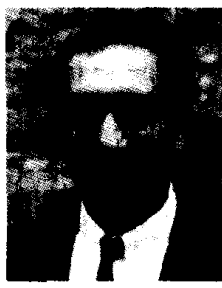

Kaveh Pahlavan was born in Teheran, Iran, on March 16, 1951. He received the M.S. degree from the University of Teheran, Iran, in 1975, and the $\mathrm{Ph} . \mathrm{D}$. degree from the Worcester Polytechnic Institute, Worcester, MA, in 1979.

From 1979 to 1983, he was an Assistant Professor in the Electrical and Computer Engineering Department at Northeastern University, Boston, MA. He was consulting with CNR, Incorporated, Needham. MA, and GTE Laboratories, Waltham, MA, during the same period. He joined Infinet Incorporated in 1983 and served as the Senior Staff Engineer and later as the Director of Advanced Developments until 1985. During this period, he was teaching DSP courses at the graduate school of Northeastern University. In September 1985 he joined the faculty of Worcester Polytechnic Institute where he is currently a Professor of Electrical Engineering and the Director of the Center for Wireless Information Network Studies (CWINS). During services at WPI, he has been a Consultant to GTE Laboratories, Waltham, MA, Steinbrecher Associates, Woban MA, Techman Int, Charlton, MA, Simplex Incorporated, Gardner, MA and WINDATA Incorporated, Northborough, MA in various aspects of the wireless indoor networks. His present areas of interest are all related to wireless information networks. His background research is on voice-band data communications and fading channel communications. He has contributed to numerous papers, two patents, and a chapter of a book, and has given tutorial talks and short courses in various countries. He was the Founder and the Chairman of the program for the IEEE Workshop on Wireless Local Area Network, held at WPI on May 9-10, 1991. He was the Organizer and the Technical Program Chairman of the IEEE, 3rd Symposium on Portable, Indoor and Mobile Radio Communications, Boston, MA, Oct. 19-21, 1992.

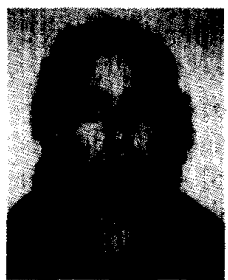

Timothy J. Holt received the B.S. and M.S. degrees in electrical engineering from Worcester Polytechnic Institute, Worcester, MA, in 1990 and 1992 respectively. As a graduate student at WPI, his research involved using ray tracing techniques to study indoor radio propagation.

In 1992, he joined Hewlett-Packard's Roseville Networks Division to begin work on a wireless network product. He is currently involved in ASIC design for $100 \mathrm{Mbps}$ copper networks. 